OPEN ACCESS

Edited by:

C. Charles Gu,

Washington University in St. Louis,

United States

Reviewed by:

Tesfaye B. Mersha,

Cincinnati Children's Hospital Medical

Center, United States

Kenneth M. Weiss,

The Pennsylvania State University,

United States

${ }^{*}$ Correspondence:

Melinda C. Aldrich

melinda.aldrich@vumc.org

Dana C. Crawford

dcc64@case.edu;

dana.crawford@case.edu

Specialty section:

This article was submitted to Applied Genetic Epidemiology,

a section of the journal

Frontiers in Genetics

Received: 30 November 2018

Accepted: 18 April 2019

Published: 10 May 2019

Citation:

Hollister BM, Farber-Eger E, Aldrich MC and Crawford DC (2019) A Social Determinant of Health May Modify Genetic Associations for Blood Pressure: Evidence From a SNP by Education Interaction in an African

American Population.

Front. Genet. 10:428.

doi: 10.3389/fgene.2019.00428

\section{A Social Determinant of Health May Modify Genetic Associations for Blood Pressure: Evidence From a SNP by Education Interaction in an African American Population}

\author{
Brittany M. Hollister ${ }^{1}$, Eric Farber-Eger ${ }^{2}$, Melinda C. Aldrich ${ }^{3 *}$ and Dana C. Crawford ${ }^{4 *}$ \\ ${ }^{1}$ Social and Behavioral Research Branch, National Human Genome Research Institute, National Institutes of Health, \\ Bethesda, MD, United States, ${ }^{2}$ Vanderbilt Institute for Clinical and Translational Research, Vanderbilt University Medical \\ Center, Nashville, TN, United States, ${ }^{3}$ Department of Thoracic Surgery, Vanderbilt Genetics Institute, Vanderbilt University \\ Medical Center, Nashville, TN, United States, ${ }^{4}$ Department of Population and Quantitative Health Sciences, Cleveland \\ Institute for Computational Biology, Case Western Reserve University, Cleveland, OH, United States
}

African Americans experience the highest burden of hypertension in the United States compared with other groups. Genetic contributions to this complex condition are now emerging in this as well as other populations through large-scale genomewide association studies (GWAS) and meta-analyses. Despite these recent discovery efforts, relatively few large-scale studies of blood pressure have considered the joint influence of genetics and social determinants of health despite extensive evidence supporting their impact on hypertension. To identify these expected interactions, we accessed a subset of the Vanderbilt University Medical Center (VUMC) biorepository linked to de-identified electronic health records (EHRs) of adult African Americans genotyped using the lllumina Metabochip $(n=2,577)$. To examine potential interactions between education, a recognized social determinant of health, and genetic variants contributing to blood pressure, we used linear regression models to investigate twoway interactions for systolic and diastolic blood pressure (DBP). We identified a two-way interaction between rs6687976 and education affecting DBP ( $p=0.052)$. Individuals homozygous for the minor allele and having less than a high school education had higher DBP compared with (1) individuals homozygous for the minor allele and high school education or greater and (2) individuals not homozygous for the minor allele and less than a high school education. To our knowledge, this is the first EHR -based study to suggest a gene-environment interaction for blood pressure in African Americans, supporting the hypothesis that genetic contributions to hypertension may be modulated by social factors.

Keywords: electronic health records, social determinants of health, African Americans, blood pressure, geneenvironment, education 


\section{INTRODUCTION}

African Americans have a higher prevalence of hypertension, or chronically high blood pressure, compared with other racial/ethnic groups (Yoon et al., 2015; Writing Group Members et al., 2016). Despite this higher burden of disease in African Americans, early genome-wide association studies (GWAS) for hypertension and systolic blood pressure (SBP) and diastolic blood pressure (DBP) were limited to populations of Europeandescent (Levy et al., 2009; Newton-Cheh et al., 2009; Wang et al., 2009; International Consortium for Blood Pressure GenomeWide Association Studies et al., 2011) or east Asian-descent (Kato et al., 2011). More recent GWAS have been performed in ancestrally diverse populations, including African Americans or African-descent populations (Adeyemo et al., 2009; Zhu et al., 2011, 2015; Kidambi et al., 2012; Franceschini et al., 2013; Hoffmann et al., 2017; Liang et al., 2017). Collectively, these associated common variants explain 3-6\% of the variance for SBP and DBP, and in the largest European-descent study to date account for up to $27 \%$ of the estimated single nucleotide polymorphism (SNP)-wide heritability for these traits (Evangelou et al., 2018).

Current GWAS findings explain only a proportion of the expected contribution from additive genetic effects. Previous twin and family studies estimate these traits have moderate to high heritability (30-70\%) (Fagard et al., 1995; Rotimi et al., 1999; Levy et al., 2000; Hottenga et al., 2005; Kupper et al., 2005), suggesting that additional genetic associations have yet to be discovered. Given that GWAS identify common single nucleotide variants (SNVs) for association, additional genetic associations may be found among rare SNVs (Doris, 2011; Russo et al., 2018). Importantly, most GWAS consider only main effects and do not consider interactions with relevant environmental exposures. Two recent and large GWAS of blood pressure have considered alcohol consumption (Feitosa et al., 2018) and smoking (Sung et al., 2018), both of which identified novel putative associations for these traits.

Here, we examine the modifying effects of education, a measure of socioeconomic status (SES) and recognized social determinant of health, on SBP and DBP traits among African Americans drawn from a clinical setting. Previous epidemiologic studies suggest that in addition to alcohol consumption and smoking, social environment and specifically SES has a strong influence on blood pressure and hypertension (Seeman et al., 2008; Cha et al., 2012; Non et al., 2012). Further, a GWAS in the Framingham Heart Study accounting for educational attainment identified novel associations for blood pressure traits among European Americans (Basson et al., 2014). Based on these prior findings, we hypothesized that educational attainment modifies associations between genetic variants and blood pressure among African Americans. To test this hypothesis, we accessed a large biobank linked to electronic health records (EHRs) in a racially diverse clinical population. We identified two associated SNPs, ARHGAP22 rs4593967 (SBP) and IQCK rs950928 (DBP), neither of which has been previously associated with blood pressure. We also identified a novel SNP-education interaction affecting DBP, suggesting social determinants of health may modify genetic effects contributing to complex human traits.

\section{MATERIALS AND METHODS}

\section{Study Population and Data Collection}

The study population is derived from BioVU, a DNA biobank of the Vanderbilt University Medical Center (VUMC) linked to de-identified EHRs. DNA samples are extracted from discarded blood samples drawn for routine clinical care (Roden et al., 2008). These samples are linked to the Synthetic Derivative (SD), the de-identified version of the VUMC EHR. Medical records within the SD are scrubbed of all Health Insurance Portability and Accountability Act (HIPAA) identifiers. This study was approved by the Vanderbilt University Institutional Review Board.

The study population consists of African American adults $>18$ years old drawn from a larger study of minority patients with DNA samples in BioVU $(n=15,863)$ (Crawford et al., 2015). We extracted relevant demographic variables, including race/ethnicity, sex, and age at data extraction available in the SD. Smoking status was extracted using International Classification of Diseases, Ninth Revision, Clinical Modification (ICD-9-CM) tobacco use codes as previously described (Wiley et al., 2013). Education was extracted from the free text of EHRs using a recently validated text-mining algorithm (Hollister et al., 2016). Education was modeled as a categorical variable: less than high school, high school, and some college or above. All weight and height measures were extracted from the EHR, and after extensive quality control, as described in Goodloe et al. (2017), median values were used to represent individual-level body mass index (BMI).

The median value of all blood pressure measurements within an individual's EHR prior to a recording of blood pressure-altering medications in the patient's medication list were used in analyses. Medications included in the keyword list of anti-hypertensives were angiotensin converting enzyme inhibitors, angiotensin II receptor blocker, beta blockers, non-dihydropyridine calcium channel blockers, hydralazine, Minoxidil, central alpha antagonists, direct renin antagonists, aldosterone antagonists, alpha antagonists, and diuretics including thiazides, K-sparing, and loop diuretics. Any blood pressure measurement found after any mention of these types of medications were excluded from analyses.

\section{Genotyping and Quality Control}

Genotyping of 15,863 DNA samples from non-European descent individuals was performed using the Metabochip, a custom Illumina genotyping array designed to target SNPs and surrounding genomic regions associated with metabolic traits and cardiovascular disease (Buyske et al., 2012; Voight et al., 2012). We restricted the following quality control and statistical analyses to DNA samples from African Americans in BioVU $(n=11,301)$. All genotyping quality control was performed using PLINK 1.9 (Chang et al., 2015). After the removal of SNPs with a minor allele frequency of less than $5 \%$, SNPs with a Hardy-Weinberg Equilibrium exact test $p$-value of less than 
$1 \times 10^{-7}$, and SNPs with a genotyping call rate of less than $95 \%$, a total of 115,834 variants remained (Supplementary Figure S1). We further removed 967 samples for either ambiguous sex, missing genotypes $(>5 \%$ ), or relatedness (twins, full siblings, parent/offspring) (Supplementary Figure S1). A total of 10,334 DNA samples passed genotyping quality control. After quality control, global ancestry was estimated using unsupervised ADMIXTURE analysis, assuming $K=2$ (Alexander et al., 2009). Linkage disequilibrium $\left(r^{2}\right)$ was calculated using 1000 Genomes Phase 3 data and an expectation-maximization algorithm adapted from Haploview (Barrett et al., 2005) available through rAggr (Edlund et al., 2017).

Local ancestry for rs6687976 was assigned as previously described (Fish et al., 2018). Briefly, SHAPEITv2 (Delaneau et al., 2013) and the 1000 Genomes Phase 3 reference panel ${ }^{1}$ were used to phase the genotype data. RFMix (Maples et al., 2013) was used to assign local ancestry. Phased chromosomal haplotypes were matched to Yoruba and CEPH/European ancestral population panels from 1000 Genomes.

\section{Statistical Analysis}

Inclusion criteria included African American adults with available Metabochip genotyping data and complete information on age, sex, BMI, premedication SBP, premedication DBP, smoking status, and education level. A total of 2,577 African Americans met genotyping quality control and had relevant covariates (Supplementary Figure S2). All statistical analyses were performed using PLINK 1.9 (Chang et al., 2015) or R ( $\mathrm{R}$ Core Team, 2008). Linear regression models were used to identify genetic variants associated with either premedication SBP or premedication DBP. A Bonferroni adjusted $p$-value of $4.32 \times 10^{-7}$ was used to determine significance. A main effects model included covariates for age, age squared, sex, BMI, smoking status, and percent global African ancestry:

$$
\begin{aligned}
\text { Premedication SBP or DBP }= & \beta_{0}+\beta_{\text {cov }}{ }^{*} X_{\text {cov }}+ \\
& \beta_{1}{ }^{*} S N P+e
\end{aligned}
$$

A second main effects model included the same covariates, but also included education. To examine the interaction between genetic variants and education and how it may affect blood pressure, we modeled two-way interactions using a linear regression model and the same covariates as in our main effects model:

$$
\begin{array}{r}
\text { Premedication SBP or DBP }=\beta_{0}+\beta_{\text {cov }}{ }^{*} X_{\text {cov }}+\beta_{1}{ }^{*} S N P+ \\
\beta_{2}{ }^{*} \text { Education }+\beta_{3}{ }^{*} S N P^{*} \text { Education }+e
\end{array}
$$

The decision was made to focus on a set of SNPs which had a $p$-value of less than $1.4 \times 10^{-5}$ from the main effects model to reduce issues with multiple testing. This significance threshold was chosen based on a Bonferroni correction for the number of SNPs that would remain if SNPs with an $r^{2}$-value of greater than 0.1 were removed from our dataset. For this set of significant SNPs, we used a model which included the main effects of

\footnotetext{
${ }^{1}$ https://mathgen.stats.ox.ac.uk/impute/impute_v2.html\#reference
}

education and the SNP, as well as the interaction term between education and the genetic variants. The significance threshold for the interaction models was based on the number of SNPs tested for association with premedication SBP and DBP $(p<0.01$ and $p<0.003$, respectively).

\section{RESULTS}

\section{Population Characteristics}

The final study population for analysis included 2,577 African American adults with Metabochip genotyping data and complete phenotype data (Supplementary Figures S1, S2). Among this study population, the majority were female $(71 \%)$ with a median age of 38 years and median BMI of $26.8 \mathrm{~kg} / \mathrm{m}^{2}$ (Table 1). Compared with a larger African American BioVU population genotyped on the Metabochip (Crawford et al., 2015), this subset had proportionally more females, was younger, and had a lower median BMI. The median premedication SBP and DBP were within the normal clinical range (122 and $74 \mathrm{mmHg}$, respectively) and most of the population was never smokers (87\%; Table 1). The median percent global African ancestry was $81.7 \%$. The majority of participants had at least a high school degree (Table 1). The median premedication SBP and DBP in this final study sample were statistically different $(p<0.05)$ from the larger study sample missing education data in the EHR but varied by only $3 \mathrm{mmHg}$

\begin{tabular}{|c|c|}
\hline Characteristic & $\begin{array}{c}\text { Number of } \\
\text { individuals } \\
n=2,577\end{array}$ \\
\hline \multicolumn{2}{|l|}{ Sex } \\
\hline Male & $753(29 \%)$ \\
\hline Female & $1,824(71 \%)$ \\
\hline Median age in years $( \pm S D)$ & $38.2( \pm 15.4)$ \\
\hline Median percent global African ancestry (range) & $81.7 \%(1.0-99.9)$ \\
\hline \multicolumn{2}{|l|}{ Education level } \\
\hline Less than high school & $328(12.7 \%)$ \\
\hline High school degree or equivalent & $1518(58.9 \%)$ \\
\hline At least some college & $731(28.4 \%)$ \\
\hline \multicolumn{2}{|l|}{ Smoking Status } \\
\hline Never smokers & $2,242(87 \%)$ \\
\hline Ever smokers & $335(13 \%)$ \\
\hline Median body mass index (kg/m²; $\pm \mathrm{SD})$ & $26.8( \pm 6.8)$ \\
\hline Median premedication systolic blood pressure (mmHg; $\pm \mathrm{SD})$ & $122.0( \pm 13.6)$ \\
\hline Median Premedication diastolic blood pressure (mmHg; \pm SD) & $74.4( \pm 9.0)$ \\
\hline
\end{tabular}
(Supplementary Table S1).

TABLE 1 | Study population characteristics representing African American adults from a biobank with electronic health record (EHR)-extracted blood pressure.

The population in this study was a subset of African Americans from the Vanderbilt University Medical Center (VUMC) biobank, BioVU. Samples were drawn from BioVU in 2011. All individuals had Metabochip genotype data which passed quality control measures. Individuals also had complete phenotype data which included age, sex, education level, smoking status, median body mass index (BMI), median premedication systolic blood pressure (SBP), and median diastolic blood pressure $(D B P)$. These phenotypes were derived from the electronic health record. African ancestry was determined using ADMIXTURE. SD, standard deviation. 


\section{Predictors of Systolic and Diastolic Blood Pressure}

In univariate analyses (Table 2), both premedication SBP and DBP were significantly associated with increasing age, male sex, and increasing BMI. SBP increased with age and DBP increased with age until around the age of 60 , then began decreasing (Supplementary Figures S3, S4).

Neither premedication SBP nor premedication DBP was associated with smoking status or global African ancestry. Also, education was not significantly associated with either premedication SBP or premedication DBP (Table 2 and Supplementary Figures S5, S6). Of all the variables tested, age and premedication DBP significantly co-varied with education (Supplementary Table S2).

\section{Education as a Modifier of Genetic Associations With Systolic and Diastolic Blood Pressure}

To test for possible interactive effects between education and genetic variants associated with SBP and DBP, we examined three models: (1) initial single SNP tests of association without education as a covariate, (2) single SNP tests of association with education as a covariate, and (3) single SNP tests of association with SNP $\times$ education interaction terms. In the first model, single SNP tests of association were performed for SBP and DBP using linear regression adjusting for age, age squared, sex, BMI, smoking status, and percent global African ancestry. For both SBP (Supplementary Figure S7) and DBP (Supplementary Figure S8), only a single SNP was statistically significant using a Bonferroni correction $\left(p<4.32 \times 10^{-7}\right)$ : ARHGAP22 rs4593967 and IQCK rs950928, respectively.
The second set of models included education in addition to other relevant covariates (Supplementary Figures S9, S10). The addition of education to the model did not change the most significantly associated SNPs for either SBP or DBP (Table 3). In the regression model for SBP that included education, rs4593967 again passed Bonferroni correction $\left(p<4.32 \times 10^{-7}\right)$, and two other SNPs (rs10921895 and rs3804485) were associated at a suggestive significance threshold $\left(p<7.24 \times 10^{-6}\right)$. For DBP, rs950928 and rs8056711 passed Bonferroni correction. However, these SNPs have the same effect size and are in perfect linkage disequilibrium $\left(r^{2}=1.0\right)$, so they likely represent the same association.

In the final set of models, education $\times$ SNP interaction terms were examined using SNPs associated with SBP or DBP at $p<1.4 \times 10^{-5}$, as described above. No interaction terms met a strict Bonferroni correction (Supplementary Figures S11, S12). However, we identified a potential SNP-education interaction affecting DBP, rs6687976 ( $p=0.052$; Table 4). This potential interaction remained with the addition of local ancestry to the model. Individuals homozygous for the minor allele and having less than a high school education had higher DBP compared with (1) individuals homozygous for the minor allele and high school education or greater and (2) individuals not homozygous for the minor allele and less than a high school education (Supplementary Figure S13). No statistically significant interactions were identified for SBP (Table 4).

\section{DISCUSSION}

We sought to determine if education, a measure of SES and a recognized social determinant of health, modified genetic

TABLE 2 | Univariate analyses between relevant covariates and median premedication blood pressure values among African American adults.

\begin{tabular}{|c|c|c|c|c|}
\hline \multirow[t]{2}{*}{ Variable } & $\begin{array}{l}\text { Effect estimate, } \beta \\
\text { (standard error) }\end{array}$ & $p$-value & $\begin{array}{c}\text { Effect estimate, } \beta \\
\text { (standard error) }\end{array}$ & $p$-value \\
\hline & \multicolumn{2}{|c|}{ Premedication SBP } & \multicolumn{2}{|c|}{ Premedication DBP } \\
\hline \multicolumn{5}{|l|}{ Education level } \\
\hline Less than high school & REF & & REF & \\
\hline High school degree and equivalent & $0.16(0.83)$ & 0.84 & $1.95(0.54)$ & $0.0003^{*}$ \\
\hline \multicolumn{5}{|l|}{ Sex } \\
\hline Male & REF & & REF & \\
\hline Female & $-4.52(0.52)$ & $<0.0001^{*}$ & $-1.48(0.35)$ & $0.0001^{*}$ \\
\hline Median BMI (kg/m²) & $0.38(0.04)$ & $<0.0001^{*}$ & $0.25(0.02)$ & $<0.0001^{*}$ \\
\hline \multicolumn{5}{|l|}{ Smoking status } \\
\hline
\end{tabular}

Prior to genetic analyses, covariates were examined to determine their association with the outcomes, premedication systolic (SBP) and diastolic blood pressure (DBP) in the study population, a subset of African Americans drawn from the Vanderbilt University Medical Center biobank BioVU ( $n=2,577)$. Each linear regression model had either median premedication SBP or median premedication DBP as the outcome. The covariates included in each model were education level, median age, sex, body mass index (BMI), smoking status, and global African ancestry. Both premedication SBP and DBP were significantly associated with age, sex, and BMI. Premedication $D B P$ is also significantly associated with education level. The symbol "** indicates statistical significance. 
TABLE 3 | Characteristics of single nucleotide polymorphisms (SNPs) associated with premedication systolic and diastolic blood pressure with and without education in the model.

\begin{tabular}{|c|c|c|c|c|c|c|c|c|c|c|}
\hline \multirow[b]{2}{*}{ SNP } & \multirow[b]{2}{*}{ Trait } & \multirow[b]{2}{*}{ Location } & \multirow[b]{2}{*}{ Gene } & \multirow[b]{2}{*}{ MAF } & \multicolumn{3}{|c|}{ Without education } & \multicolumn{3}{|c|}{ With education } \\
\hline & & & & & $\begin{array}{c}\text { Effect } \\
\text { estimate }\end{array}$ & $\begin{array}{l}\text { Standard } \\
\text { error }\end{array}$ & $p$-value & $\begin{array}{l}\text { Effect } \\
\text { estimate }\end{array}$ & $\begin{array}{l}\text { Standard } \\
\text { error }\end{array}$ & $p$-value \\
\hline rs4593967 & SBP & Intron & ARHGAP22 & 0.14 & -2.51 & 0.46 & $1.45 \times 10^{-7}$ & -2.53 & 0.48 & $1.16 \times 10^{-7}$ \\
\hline rs10921895 & SBP & Intergenic & & 0.37 & -1.52 & 0.37 & $5.31 \times 10^{-6}$ & -1.55 & 0.36 & $3.92 \times 10^{-6}$ \\
\hline rs3804485 & SBP & Intron & LY86 & 0.41 & 1.48 & 0.32 & $7.11 \times 10^{-6}$ & 1.51 & 0.33 & $5.20 \times 10^{-6}$ \\
\hline rs950928 & DBP & Intron & IQCK & 0.36 & -1.04 & 0.24 & $3.13 \times 10^{-6}$ & -1.10 & 0.22 & $4.53 \times 10^{-7}$ \\
\hline rs8056711 & DBP & Intron & IQCK & 0.36 & -1.04 & 0.24 & $3.13 \times 10^{-6}$ & -1.10 & 0.22 & $4.53 \times 10^{-7}$ \\
\hline
\end{tabular}

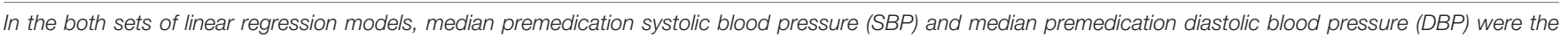

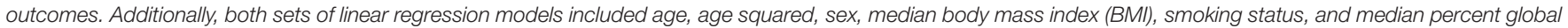

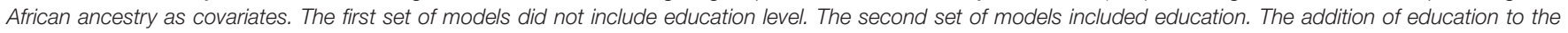
model did not change which SNPS were most associated with SBP or DBP. Bolded p-values are considered statistically significant after Bonferroni correction.

TABLE 4 | Single nucleotide polymorphisms (SNPS) examined for interactions with education level impacting median premedication systolic and diastolic blood pressure.

\begin{tabular}{|c|c|c|}
\hline $\begin{array}{l}\text { SNP in education } \\
\text { interaction term }\end{array}$ & $\begin{array}{l}p \text {-value for the } \\
\text { interaction between high } \\
\text { school and SNP }\end{array}$ & $\begin{array}{c}p \text {-value for } \\
\text { interaction between } \\
\text { college and SNP }\end{array}$ \\
\hline \multicolumn{3}{|c|}{ Systolic Blood Pressure } \\
\hline rs4593967_A & 0.886 & 0.858 \\
\hline rs10921895_G & 0.896 & 0.746 \\
\hline rs3804485_C & 0.260 & 0.863 \\
\hline rs11066700_A & 0.178 & 0.200 \\
\hline \multicolumn{3}{|c|}{ Diastolic Blood Pressure } \\
\hline rs950928_G & 0.175 & 0.298 \\
\hline rs8056711_C & 0.175 & 0.297 \\
\hline rs9931167_A & 0.927 & 0.503 \\
\hline rs11648873_T & 0.940 & 0.478 \\
\hline rs28681321_A & 0.941 & 0.461 \\
\hline rs6687976_A & 0.052 & 0.996 \\
\hline chr16.19642355_G & 0.094 & 0.110 \\
\hline rs3095994_A & 0.738 & 0.726 \\
\hline rs1273518_G & 0.218 & 0.648 \\
\hline rs1858975_A & 0.076 & 0.091 \\
\hline rs4593967_A & 0.512 & 0.768 \\
\hline rs8046628_C & 0.181 & 0.316 \\
\hline rs6497402_A & 0.062 & 0.085 \\
\hline chr16.19641087_A & 0.064 & 0.076 \\
\hline
\end{tabular}

Median premedication systolic blood pressure (SBP) and diastolic blood pressure (DBP) were outcomes in the linear regression models. Covariates included in the models were age, age squared, sex, body mass index, smoking status, and African ancestry. The main effect of education and the SNP, as well as the SNP $\times$ education interaction term were also included in the model. Less than high school was the reference group within the regression models. The $p$-value for the potential SNPeducation interaction is bolded.

associations with SBP and DBP in African Americans. A previous study suggested gene $\times$ education interactions occur with blood pressure, but this study was conducted in a Europeandescent population (Basson et al., 2014). Associations between premedication SBP or premedication DBP and genetic variants from the Metabochip were examined, while including known predictors of blood pressure (age, BMI, sex, percent African ancestry, and smoking status) in the model. Results were compared with models which included a main effect for education, and a main effect for education plus a SNP-education interaction term. We observed a suggestive SNP by education interaction affecting DBP, a result not explained by local genetic ancestry. This potential interaction requires statistical replication and further investigation.

\section{Models Without Interaction}

In univariate analyses the associations between premedication SBP and DBP and increasing age, male sex as well as increasing BMI were consistent with previous reports (August, 1999; Wright et al., 2011; Dua et al., 2014). The patterns of associations between SBP and DBP and age across the age continuum are also consistent with previous reports (Liang et al., 2017; Evangelou et al., 2018).

Intronic ARHGAP22 rs4593967 was significantly associated with SBP and has not been previously reported as associated with blood pressure or hypertension. The minor allele frequency for ARHGAP22 rs4593967 in this African American sample was 0.14 , consistent with frequencies reported for Africandescent populations included in The Genome Aggregation Database (0.148; Lek et al., 2016) and the 1000 Genomes Project (0.176; 1000 Genomes Project Consortium et al., 2015). Conversely, the minor allele is less frequently observed among populations of European $(\sim 0.08)$ or East Asian-descent $(<0.01)$. No other common (MAF > 1\%) variants within $500 \mathrm{~kb}$ are in strong linkage disequilibrium $\left(r^{2} \geq 0.80\right)$ with $r 54593967$ in African-descent populations from the 1000 Genomes Project. ARHGAP22 encodes the rho GTPase activating protein 22 and is widely expressed with highest expression levels in the brain. Variants within ARHGAP22 have been associated with diabetic retinopathy, conduct disorder, daytime sleep, and selfemployment (Dick et al., 2011; Huang et al., 2011; Van Der Loos et al., 2013; Spada et al., 2016), but these associations have not been replicated.

Intronic IQCK rs950928 was significantly associated with DBP after adjusting for multiple testing. Like ARHGAP22 rs4593967, the minor allele frequency for IQCK rs950928 is 
higher among populations of African-descent $(\sim 0.40)$ compared with European-descent populations $(\sim 0.15)$. IQCK rs950928 is in perfect or strong linkage disequilibrium with rs8056711 and rs59009734 in African-descent populations, neither of which has been previously associated with human disease or traits. $I Q C K$, which overlaps with several genes including KNOP1, encodes for IQ motif containing $K$ and serves as an EF hand protein binding site. Like ARHGAP22, IQCK is highly expressed in the brain. A search within the Genotype-Tissue Expression (GTEx Consortium, 2013) database suggests that both rs8056711 and rs59009734 may be expression quantitative loci (eQTL), where each addition of the minor allele is associated with higher gene expression for several tissues including the right atrium auricular region of the heart and the aorta. While IQCK rs950928 and its associated SNPs rs8056711 and rs59009734 have not been previously associated with any phenotypes, common variants within IQCK have previously been associated with blood pressure, BMI, bone density, heart rate, chronic obstructive pulmonary disease, bipolar disorder, and a BMIeducation interaction (Cho et al., 2009; Liu et al., 2010; Wan et al., 2011; Boardman et al., 2014; Winham et al., 2014).

Despite the present study's small sample size $(n=2,577)$, there was sufficient power $(80 \%)$ to detect significant associations with moderate effect size of 1.0 and a minor allele frequency of 0.20 . For less common variants $(\mathrm{MAF}=0.10)$, the study was powered to detect alleles with an effect size of 1.5 or greater. For variants with a MAF of 0.05 , an effect size of 2.0 was needed in order to detect the variant's effect. This study was not powered to detect any of the variants reported in the recent one million-person GWAS of blood pressure, as the variant with the largest effect size in that study was less than 1.0, with a median effect size of $0.219 \mathrm{mmHg}$ (Evangelou et al., 2018). The limited power due to small sample size and limited directly genotyped variants likely contributed to the lack of replication of SNPs known to be associated with blood pressure in African Americans from previous GWAS.

\section{SNP $\times$ Education Interactions}

We identified a possible SNP-education interaction affecting DBP for rs6687976 $(p=0.052)$. As the addition of local ancestry to the model did not alter the association, we expect that this observation is a result of true modifying effects of SES rather than ancestry. Individuals with two minor alleles and less than a high school education had higher blood pressure compared to those with two minor alleles and a high school education or those with less than a high school education and fewer minor alleles (Supplementary Figure S13). SNP rs6687976 is located within an intergenic region of chromosome 1 (Chr1:105674536 in GRCh37.p13) and has not been previously associated with any human traits within the literature. It is also not identified as an eQTL in GTEx (GTEx Consortium, 2013). Despite the limited information known about rs6687976, this result suggests that interactions between markers of social determinants of health and genetic variants affecting blood pressure likely exist, consistent with the findings of other studies that have observed interactions between genetic variants and social factors such as depression (Smith et al., 2017), perceived discrimination (Taylor et al., 2017), and cigarette smoking (Taylor et al., 2016).

\section{Limitations}

The present study has several limitations. Primarily, the sample size is limited driven by the inclusion criteria of complete phenotype data for a specific racial/ethnic group within the larger clinical dataset. Therefore, we are unable to detect any variants of smaller effect sizes. The requirement for complete data may have also introduced biases that limit the interpretation and generalizability of these data.

In addition to the limited sample size, the study population was also different compared with previously published studies of blood pressure in African American populations. While the proportion of females, median BMI, percent African ancestry, median SBP, and median DBP were comparable with previous studies (Parra et al., 1998; Dumitrescu et al., 2015; Baharian et al., 2016; Franceschini et al., 2016; Jones et al., 2018; Restrepo et al., 2018), the population in this study did have a much lower median age, over 15 years younger. Given that blood pressure increases with age, this younger study population may have reduced variability in blood pressure measurements compared with the older published study populations with right-skewed distributions (Wright et al., 2011).

Another limitation was the lack of a replication dataset; therefore, all associations reported here are putative pending statistical replication or corroborative functional data. To date, other studies comparable or larger in sample size have not yet reported associations between these SNPs and blood pressure (Hoffmann et al., 2017). Furthermore, the genotyping array used here was also designed to include rare variation collected from the African ancestry samples as part of the 1000 Genomes Project. Therefore, many of the variants on the Metabochip were rare in African ancestry populations (Buyske et al., 2012) and filtered out during the quality control process as the present study was not powered to detect associations for rare variation.

There were also limitations regarding the phenotype data. All the variables were extracted from EHRs. While these records have extensive amounts of data, the data recorded by healthcare providers are not always accurate and the ability to extract the data can be limited. Furthermore although the positive predictive value of our algorithm was $80 \%$ (Hollister et al., 2016), there may have been inaccurate education information for the individuals within the dataset.

Determining which blood pressure measurements to use in the study is also a challenge, as measurements can vary widely across the EHR. The median blood pressure measurements were chosen for our study to reduce the influence of this variation. Beyond the inaccuracies and decisions to be made regarding the information within the EHR, blood pressure is difficult to measure within the clinic. Measurements of blood pressure can vary due to the calibration of instruments, the time of day it is measured, and due to illness (Jones et al., 2003). Patients also tend to have higher blood pressure within a clinical setting due to stress (Jones et al., 2003). To avoid these potential biases as much as possible, we chose median premedication blood pressure 
values for analysis, thereby avoiding outlier measurements and the changes introduced by blood pressure medications.

Finally, while education is a recognized social determinant of health, it is not a perfect proxy for social experiences. Still, evidence suggests that educational attainment can be a reflection of earning potential and social status (Shavers, 2007; Tamborini et al., 2015). Education has been shown to be associated with life expectancy, numerous biomarkers, and other health outcomes such as obesity and smoking (Seeman et al., 2008; National Center for Health Statistics, 2012). Low educational attainment itself is not the cause of poor health outcomes, but rather a variable often associated with individual-level behavioral determinants (e.g., smoking) or community-level determinants (e.g., racial segregation) that may influence blood pressure. Neither of these determinants is routinely recorded with the EHR; in contrast, educational attainment is often mentioned in the EHR. The availability of these data coupled with the observation that individual educational attainment is often stable over time make this variable a robust albeit imperfect proxy for social experiences.

\section{Strengths}

Despite the limitations within the study, there were also several strengths. Primarily, this is the first study to incorporate EHR-derived education information into a large-scale genetic investigation. This study is a proof of principle that EHR-derived social determinant information can be investigated in a GWAS setting, thus breaking new ground to incorporate social factors in genetic studies among biobank populations. This is also the first analysis to observe an interaction between education and a common genetic variant with blood pressure in an African American population.

Despite the consistent association between social environment and health, social determinants of health are typically not included in genetic studies of health outcomes. For studies that access biobanks, the lack of social determinant data is likely related to the difficulty in accessing these data within the EHR, where they are not usually recorded in structured fields. The algorithms used in our study are the first to extract these important data from EHRs for research purposes (Hollister et al., 2016).

This study paves the road for the incorporation of education, as well as other social determinants of health, into genetic studies using biobank populations. The SNP-by-education interaction we observed affecting DBP (rs6687976) could suggest an example of a possible biological impact of the adversity experienced due to lower educational achievement. Only individuals homozygous for the minor allele who had less than a high school education experienced an increase in DBP. This association needs to

\section{REFERENCES}

1000 Genomes Project Consortium, Auton, A., Brooks, L. D., Durbin, R. M., Garrison, E. P., Kang, H. M., et al. (2015). A global reference for human genetic variation. Nature 526, 68-74. doi: 10.1038/nature15393

Adeyemo, A., Gerry, N., Chen, G., Herbert, A., Doumatey, A., Huang, H., et al. (2009). A genome-wide association study of hypertension and blood pressure be replicated; however, it suggests a potential pathway for the biological imbedding of stress experiences (represented by lower educational attainment) affecting blood pressure and risk for hypertension. Further studies are needed to support this hypothesis. We anticipate that this research will encourage other investigators to continue to study the genetics of health outcomes associated with racial health disparities and to incorporate social determinants of health within these studies.

\section{AUTHOR CONTRIBUTIONS}

$\mathrm{BH}$ conducted the analyses and wrote manuscript. EF-E helped to extract the phenotype data from the electronic health record. MA and DC contributed to guidance on project, and manuscript writing and editing.

\section{FUNDING}

This work was supported by the National Institutes of Health (NIH) U01 HG004798 and its ARRA supplements (DC), as well as National Cancer Institute 1K07CA172294 (MA). This publication was also made possible by the Clinical and Translational Science Collaborative of Cleveland, 4UL1TR0002548, from the National Center for Advancing Translational Sciences (NCATS) component of the National Institutes of Health and NIH Roadmap for Medical Research. Its contents are solely the responsibility of the authors and do not necessarily represent the official views of the NIH. The dataset (s) used for the analyses described were obtained from Vanderbilt University Medical Center's BioVU, which is supported by institutional funding and the National Center for Research Resources, grant UL1 RR024975-01 (now at NCATS, grant 2UL1 TR000445-06).

\section{ACKNOWLEDGMENTS}

We would like to thank Drs. Alex Fish and William Bush for access to the local genetic ancestry data, and we further thank Dr. Bush for helpful comments during the revision process.

\section{SUPPLEMENTARY MATERIAL}

The Supplementary Material for this article can be found online at: https://www.frontiersin.org/articles/10.3389/fgene. 2019.00428/full\#supplementary-material

in African Americans. PLoS Genet. 5:e1000564. doi: 10.1371/journal.pgen. 1000564

Alexander, D. H., Novembre, J., and Lange, K. (2009). Fast model-based estimation of ancestry in unrelated individuals. Genome Res. 19, 1655-1664. doi: 10.1101/ gr.094052.109

August, P. (1999). Hypertension in men. J. Clin. Endocrinol. Metab. 84, 3451-3454. 
Baharian, S., Barakatt, M., Gignoux, C. R., Shringarpure, S., Errington, J., Blot, W. J., et al. (2016). The great migration and African-American genomic diversity. PLoS Genet. 12:e1006059. doi: 10.1371/journal.pgen.1006059

Barrett, J. C., Fry, B., Maller, J., and Daly, M. J. (2005). Haploview: analysis and visualization of LD and haplotype maps. Bioinformatics 21, 263-265. doi: 10.1093/bioinformatics/bth457

Basson, J., Sung, Y. J., Schwander, K., Kume, R., Simino, J., De Las Fuentes, L., et al. (2014). Gene-education interactions identify novel blood pressure loci in the Framingham Heart Study. Am. J. Hypertens. 27, 431-444. doi: 10.1093/ajh/ hpt 283

Boardman, J. D., Domingue, B. W., Blalock, C. L., Haberstick, B. C., Harris, K. M., and Mcqueen, M. B. (2014). Is the gene-environment interaction paradigm relevant to genome-wide studies? The case of education and body mass index. Demography 51, 119-139. doi: 10.1007/s13524-013-0259-4

Buyske, S., Wu, Y., Carty, C. L., Cheng, I., Assimes, T. L., and Dumitrescu, L. (2012). Evaluation of the metabochip genotyping array in African Americans and implications for fine mapping of GWAS-identified loci: the PAGE study. PLoS One 7:e35651. doi: 10.1371/journal.pone.0035651

Cha, S. H., Park, H. S., and Cho, H. J. (2012). Socioeconomic disparities in prevalence, treatment, and control of hypertension in middle-aged Koreans. J. Epidemiol. 22, 425-432. doi: 10.2188/jea.je20110132

Chang, C. C., Chow, C. C., Tellier, L. C., Vattikuti, S., Purcell, S. M., and Lee, J. J. (2015). Second-generation PLINK: rising to the challenge of larger and richer datasets. Gigascience 4:7. doi: 10.1186/s13742-015-0047-8

Cho, Y. S., Go, M. J., Kim, Y. J., Heo, J. Y., Oh, J. H., Ban, H. J., et al. (2009). A large-scale genome-wide association study of Asian populations uncovers genetic factors influencing eight quantitative traits. Nat. Genet. 41, 527-534. doi: $10.1038 /$ ng.357

GTEx Consortium. (2013). The genotype-tissue expression (GTEx) project. Nat. Genet. 45, 580-585. doi: 10.1038/ng.2653

Crawford, D. C., Goodloe, R., Farber-Eger, E., Boston, J., Pendergrass, S. A., Haines, J. L., et al. (2015). Leveraging epidemiologic and clinical collections for genomic studies of complex traits. Hum. Hered. 79, 137-146. doi: 10.1159/000381805

Delaneau, O., Zagury, J. F., and Marchini, J. (2013). Improved whole-chromosome phasing for disease and population genetic studies. Nat. Methods 10, 5-6. doi: 10.1038/nmeth.2307

Dick, D. M., Aliev, F., Krueger, R. F., Edwards, A., Agrawal, A., Lynskey, M., et al. (2011). Genome-wide association study of conduct disorder symptomatology. Mol. Psychiatry 16, 800-808. doi: 10.1038/mp.2010.73

Doris, P. A. (2011). The genetics of blood pressure and hypertension: the role of rare variation. Cardiovasc. Ther. 29, 37-45. doi: 10.1111/j.1755-5922.2010. 00246.x

Dua, S., Bhuker, M., Sharma, P., Dhall, M., and Kapoor, S. (2014). Body mass index relates to blood pressure among adults. N. Am. J. Med. Sci. 6, 89-95. doi: 10.4103/1947-2714.127751

Dumitrescu, L., Restrepo, N. A., Goodloe, R., Boston, J., Farber-Eger, E., Pendergrass, S. A., et al. (2015). Towards a phenome-wide catalog of human clinical traits impacted by genetic ancestry. Biodata Min. 8:35. doi: 10.1186/ s13040-015-0068-y

Edlund, C. K., Conti, D. V., and Van Den Berg, D. J. (2017). rAggr. Available at: http://raggr.usc.edu/ (accessed November 7, 2018).

Evangelou, E., Warren, H. R., Mosen-Ansorena, D., Mifsud, B., Pazoki, R., Gao, H., et al. (2018). Genetic analysis of over 1 million people identifies 535 new loci associated with blood pressure traits. Nat. Genet. 50, 1412-1425. doi: 10.1038/ s41588-018-0205- $\mathrm{x}$

Fagard, R., Brguljan, J., Staessen, J., Thijs, L., Derom, C., Thomis, M., et al. (1995). Heritability of conventional and ambulatory blood pressures. A study in twins. Hypertension 26, 919-924. doi: 10.1161/01.hyp.26.6.919

Feitosa, M. F., Kraja, A. T., Chasman, D. I., Sung, Y. J., Winkler, T. W., Ntalla, I., et al. (2018). Novel genetic associations for blood pressure identified via genealcohol interaction in up to $570 \mathrm{~K}$ individuals across multiple ancestries. PLoS One 13:e0198166. doi: 10.1371/journal.pone.0198166

Fish, A. E., Crawford, D. C., Capra, J. A., and Bush, W. S. (2018). Local ancestry transitions modify SNP-trait associations. Pac. Symp. Biocomput. 23, 424-435.

Franceschini, N., Carty, C. L., Lu, Y., Tao, R., Sung, Y. J., Manichaikul, A., et al. (2016). Variant discovery and fine mapping of genetic loci associated with blood pressure traits in Hispanics and African Americans. PLoS One 11:e0164132. doi: 10.1371/journal.pone.0164132
Franceschini, N., Fox, E., Zhang, Z., Edwards, T. L., Nalls, M. A., Sung, Y. J., et al. (2013). Genome-wide association analysis of blood-pressure traits in African-ancestry individuals reveals common associated genes in African and non-African populations. Am. J. Hum. Genet. 93, 545-554. doi: 10.1016/j.ajhg. 2013.07.010

Goodloe, R., Farber-Eger, E., Boston, J., Crawford, D. C., and Bush, W. S. (2017). Reducing clinical noise for body mass index measures due to unit and transcription errors in the electronic health record. AMIA Jt. Summits Transl. Sci. Proc. 2017, 102-111.

Hoffmann, T. J., Ehret, G. B., Nandakumar, P., Ranatunga, D., Schaefer, C., Kwok, P. Y., et al. (2017). Genome-wide association analyses using electronic health records identify new loci influencing blood pressure variation. Nat. Genet. 49, 54-64. doi: 10.1038/ng.3715

Hollister, B. M., Restrepo, N. A., Farber-Eger, E., Crawford, D. C., Aldrich, M. C., and Non, A. (2016). Development and performance of text-mining algorithms to extract socioeconomic status from de-identified electronic health records. Pac. Symp. Biocomput. 22, 230-241. doi: 10.1142/9789813207813_0023

Hottenga, J. J., Boomsma, D. I., Kupper, N., Posthuma, D., Snieder, H., Willemsen, G., et al. (2005). Heritability and stability of resting blood pressure. Twin Res. Hum. Genet. 8, 499-508. doi: 10.1375/183242705774310123

Huang, Y. C., Lin, J. M., Lin, H. J., Chen, C. C., Chen, S. Y., Tsai, C. H., et al. (2011). Genome-wide association study of diabetic retinopathy in a Taiwanese population. Ophthalmology 118, 642-648. doi: 10.1016/j.ophtha.2010.07.020

International Consortium for Blood Pressure Genome-Wide Association Studies, Ehret, G. B., Munroe, P. B., Rice, K. M., Bochud, M., Johnson, A. D., et al. (2011). Genetic variants in novel pathways influence blood pressure and cardiovascular disease risk. Nature 478, 103-109. doi: 10.1038/nature10405

Jones, C. C., Mercaldo, S. F., Blume, J. D., Wenzlaff, A. S., Schwartz, A. G., Chen, H., et al. (2018). Racial disparities in lung cancer survival: the contribution of stage, treatment, and ancestry. J. Thorac. Oncol. 13, 1464-1473. doi: 10.1016/j. jtho.2018.05.032

Jones, D. W., Appel, L. J., Sheps, S. G., Roccella, E. J., and Lenfant, C. (2003). Measuring blood pressure accurately: new and persistent challenges. JAMA 289, 1027-1030.

Kato, N., Takeuchi, F., Tabara, Y., Kelly, T. N., Go, M. J., and Sim, X. (2011). Meta-analysis of genome-wide association studies identifies common variants associated with blood pressure variation in east Asians. Nat. Genet. 43, 531-538. doi: 10.1038/ng.834

Kidambi, S., Ghosh, S., Kotchen, J. M., Grim, C. E., Krishnaswami, S., Kaldunski, M. L., et al. (2012). Non-replication study of a genome-wide association study for hypertension and blood pressure in African Americans. BMC Med. Genet. 13:27. doi: 10.1186/1471-2350-13-27

Kupper, N., Willemsen, G., Riese, H., Posthuma, D., Boomsma, D. I., and De Geus, E. J. (2005). Heritability of daytime ambulatory blood pressure in an extended twin design. Hypertension 45, 80-85. doi: 10.1161/01.hyp.0000149952. 84391.54

Lek, M., Karczewski, K. J., Minikel, E. V., Samocha, K. E., Banks, E., Fennell, T., et al. (2016). Analysis of protein-coding genetic variation in 60,706 humans. Nature 536, 285-291. doi: 10.1038/nature19057

Levy, D., Destefano, A. L., Larson, M. G., O’Donnell, C. J., Lifton, R. P., Gavras, H., et al. (2000). Evidence for a gene influencing blood pressure on chromosome 17. Genome scan linkage results for longitudinal blood pressure phenotypes in subjects from the Framingham Heart Study. Hypertension 36, 477-483. doi: 10.1161/01.hyp.36.4.477

Levy, D., Ehret, G. B., Rice, K., Verwoert, G. C., Launer, L. J., and Dehghan, A. (2009). Genome-wide association study of blood pressure and hypertension. Nat. Genet. 41, 677-687. doi: 10.1038/ng.384

Liang, J., Le, T. H., Edwards, D. R. V., Tayo, B. O., Gaulton, K. J., and Smith, J. A. (2017). Single-trait and multi-trait genome-wide association analyses identify novel loci for blood pressure in African-ancestry populations. PLoS Genet. 13:e1006728. doi: 10.1371/journal.pgen.1006728

Liu, J. Z., Medland, S. E., Wright, M. J., Henders, A. K., Heath, A. C., Madden, P. A., et al. (2010). Genome-wide association study of height and body mass index in Australian twin families. Twin Res. Hum. Genet. 13, 179-193. doi: 10.1375/twin.13.2.179

Maples, B. K., Gravel, S., Kenny, E. E., and Bustamante, C. D. (2013). RFMix: a discriminative modeling approach for rapid and robust local-ancestry inference. Am. J. Hum. Genet. 93, 278-288. doi: 10.1016/j.ajhg.2013.06.020 
National Center for Health Statistics (2012). Health, United States, 2011: With Special Feature on Socioeconomic Status and Health. Hyattsville, MD: National Center for Health Statistics. Available at: https://www.cdc.gov/nchs/data/hus/ hus11.pdf

Newton-Cheh, C., Johnson, T., Gateva, V., Tobin, M. D., Bochud, M., Coin, L., et al. (2009). Genome-wide association study identifies eight loci associated with blood pressure. Nat. Genet. 41, 666-676. doi: 10.1038/ng.361

Non, A. L., Gravlee, C. C., and Mulligan, C. J. (2012). Education, genetic ancestry, and blood pressure in African Americans and Whites. Am. J. Public Health 102, 1559-1565. doi: 10.2105/AJPH.2011.300448

Parra, E. J., Marcini, A., Akey, J., Martinson, J., Batzer, M. A., Cooper, R., et al. (1998). Estimating African American admixture proportions by use of population-specific alleles. Am. J. Hum. Genet. 63, 1839-1851. doi: 10.1086/ 302148

R Core Team (2008). R: A Language and Environment for Statistical Computing. Vienna: R Foundation for Statistical Computing.

Restrepo, N. A., Laper, S. M., Farber-Eger, E., and Crawford, D. C. (2018). Local genetic ancestry in CDKN2B-AS1 is associated with primary openangle glaucoma in an African American cohort extracted from de-identified electronic health records. BMC Med. Genomics 11:70. doi: 10.1186/s12920-0180392-4

Roden, D. M., Pulley, J. M., Basford, M. A., Bernard, G. R., Clayton, E. W., Balser, J. R., et al. (2008). Development of a large-scale de-identified DNA biobank to enable personalized medicine. Clin. Pharmacol. Ther. 84, 362-369. doi: 10.1038/clpt.2008.89

Rotimi, C. N., Cooper, R. S., Cao, G., Ogunbiyi, O., Ladipo, M., Owoaje, E., et al. (1999). Maximum-likelihood generalized heritability estimate for blood pressure in Nigerian families. Hypertension 33, 874-878. doi: 10.1161/01.hyp. 33.3.874

Russo, A., Di Gaetano, C., Cugliari, G., and Matullo, G. (2018). Advances in the genetics of hypertension: the effect of rare variants. Int. J. Mol. Sci. 19:E688. doi: 10.3390/ijms19030688

Seeman, T., Merkin, S. S., Crimmins, E., Koretz, B., Charette, S., and Karlamangla, A. (2008). Education, income and ethnic differences in cumulative biological risk profiles in a national sample of US adults: NHANES III (1988-1994). Soc. Sci. Med. 66, 72-87. doi: 10.1016/j.socscimed.2007.08.027

Shavers, V. L. (2007). Measurement of socioeconomic status in health disparities research. J. Natl. Med. Assoc. 99, 1013-1023.

Smith, J. A., Zhao, W., Yasutake, K., August, C., Ratliff, S. M., Faul, J. D., et al. (2017). Gene-by-psychosocial factor interactions influence diastolic blood pressure in European and African ancestry populations: meta-analysis of four cohort studies. Int. J. Environ. Res. Public Health 14:E1596. doi: 10.3390/ ijerph14121596

Spada, J., Scholz, M., Kirsten, H., Hensch, T., Horn, K., Jawinski, P., et al. (2016). Genome-wide association analysis of actigraphic sleep phenotypes in the LIFE adult study. J. Sleep Res. 25, 690-701. doi: 10.1111/jsr.12421

Sung, Y. J., Winkler, T. W., De Las Fuentes, L., Bentley, A. R., Brown, M. R., Kraja, A. T., et al. (2018). A large-scale multi-ancestry genome-wide study accounting for smoking behavior identifies multiple significant loci for blood pressure. Am. J. Hum. Genet. 102, 375-400. doi: 10.1016/j.ajhg.2018.01.015

Tamborini, C. R., Kim, C., and Sakamoto, A. (2015). Education and lifetime earnings in the United States. Demography 52, 1383-1407. doi: 10.1007/s13524015-0407-0

Taylor, J. Y., Schwander, K., Kardia, S. L., Arnett, D., Liang, J., Hunt, S. C., et al. (2016). A Genome-wide study of blood pressure in African Americans accounting for gene-smoking interaction. Sci. Rep. 6:18812. doi: 10.1038/ srep18812

Taylor, J. Y., Sun, Y. V., Barcelona De Mendoza, V., Ifatunji, M., Rafferty, J., Fox, E. R., et al. (2017). The combined effects of genetic risk and perceived discrimination on blood pressure among African Americans in the Jackson Heart Study. Medicine 96:e8369. doi: 10.1097/MD.00000000000 08369

Van Der Loos, M. J., Rietveld, C. A., Eklund, N., Koellinger, P. D., Rivadeneira, F., and Abecasis, G. R. (2013). The molecular genetic architecture of selfemployment. PLoS One 8:e60542. doi: 10.1371/journal.pone.0060542

Voight, B. F., Kang, H. M., Ding, J., Palmer, C. D., Sidore, C., and Chines, P. S. (2012). The metabochip, a custom genotyping array for genetic studies of metabolic, cardiovascular, and anthropometric traits. PLoS Genet. 8:e1002793. doi: 10.1371/journal.pgen.1002793

Wan, E. S., Cho, M. H., Boutaoui, N., Klanderman, B. J., Sylvia, J. S., and Ziniti, J. P. (2011). Genome-wide association analysis of body mass in chronic obstructive pulmonary disease. Am. J. Respir. Cell Mol. Biol. 45, 304-310. doi: 10.1165/rcmb. 2010-0294OC

Wang, Y., O’Connell, J. R., Mcardle, P. F., Wade, J. B., Dorff, S. E., Shah, S. J., et al. (2009). From the cover: whole-genome association study identifies STK39 as a hypertension susceptibility gene. Proc. Natl. Acad. Sci. U.S.A. 106, 226-231. doi: 10.1073/pnas.0808358106

Wiley, L. K., Shah, A., Xu, H., and Bush, W. S. (2013). ICD-9 tobacco use codes are effective identifiers of smoking status. J. Am. Med. Inform. Assoc. 20, 652-658. doi: 10.1136/amiajnl-2012-001557

Winham, S. J., Cuellar-Barboza, A. B., Oliveros, A., Mcelroy, S. L., Crow, S., Colby, C., et al. (2014). Genome-wide association study of bipolar disorder accounting for effect of body mass index identifies a new risk allele in TCF7L2. Mol. Psychiatry 19, 1010-1016. doi: 10.1038/mp.2013.159

Wright, J. D., Hughes, J. P., Ostchega, Y., Yoon, S. S., Nwankwo, T. (2011). Mean systolic and diastolic blood pressure in adults aged 18 and over in the United States, 2001-2008. Natl. Health Stat. Report. 35, 1-22.

Writing Group Members, Mozaffarian, D., Benjamin, E. J., Go, A. S., Arnett, D. K., and Blaha, M. J. (2016). Heart disease and stroke statistics-2016 update: a report from the American heart association. Circulation 133, e38-e360.

Yoon, S. S., Gu, Q., Nwankwo, T., Wright, J. D., Hong, Y., and Burt, V. (2015). Trends in blood pressure among adults with hypertension: United States, 2003 to 2012. Hypertension 65, 54-61. doi: 10.1161/HYPERTENSIONAHA.114. 04012

Zhu, X., Feng, T., Tayo, B. O., Liang, J., Young, J. H., Franceschini, N., et al. (2015). Meta-analysis of correlated traits via summary statistics from GWASs with an application in hypertension. Am. J. Hum. Genet. 96, 21-36. doi: 10.1016/j.ajhg. 2014.11.011

Zhu, X., Young, J. H., Fox, E., Keating, B. J., Franceschini, N., and Kang, S. (2011). Combined admixture mapping and association analysis identifies a novel blood pressure genetic locus on 5p13: contributions from the CARe consortium. Hum. Mol. Genet. 20, 2285-2295. doi: 10.1093/hmg/ ddr113

Conflict of Interest Statement: The authors declare that the research was conducted in the absence of any commercial or financial relationships that could be construed as a potential conflict of interest.

The handling Editor declared a past co-authorship with one of the authors DC.

Copyright (C) 2019 Hollister, Farber-Eger, Aldrich and Crawford. This is an openaccess article distributed under the terms of the Creative Commons Attribution License (CC BY). The use, distribution or reproduction in other forums is permitted, provided the original author(s) and the copyright owner(s) are credited and that the original publication in this journal is cited, in accordance with accepted academic practice. No use, distribution or reproduction is permitted which does not comply with these terms. 